\section{No direction known}

\section{The End of Certainty: Time, Chaos,} and the New Laws of Nature

by Ilya Prigogine

Free Press/Simon and Schuster: 1997. Pp.

228. $\$ 24, \mathfrak{£ 2 0}$

\section{In Search of Lost Time}

by Derek York

Institute of Physics Publishing: 1997. Pp. 141. $\mathfrak{E} 7.95, \$ 15$ ( $p b k$ )

\section{Huw Price}

Can physics explain the difference between past and future? The problem is that the laws of physics seem to be time-symmetric: if they allow a process with one temporal orientation, they allow it in reverse. Yet many ordinary processes seem to be irreversible. Ilya Prigogine calls this the 'time paradox', and argues that the solution lies in chaos theory and related methods pioneered by himself and his Brussels colleagues - a radical alternative, he believes, to a tradition due to Ludwig Boltzmann.

Prigogine paints with a broad brush. For example, he seems to equate explaining the thermodynamic asymmetry with finding a physical basis for the apparent flow of time. But these are different issues. Someone who treats time on a par with space, denying that there is objective flow, will still acknowledge that the Universe is asymmetric along its tem-

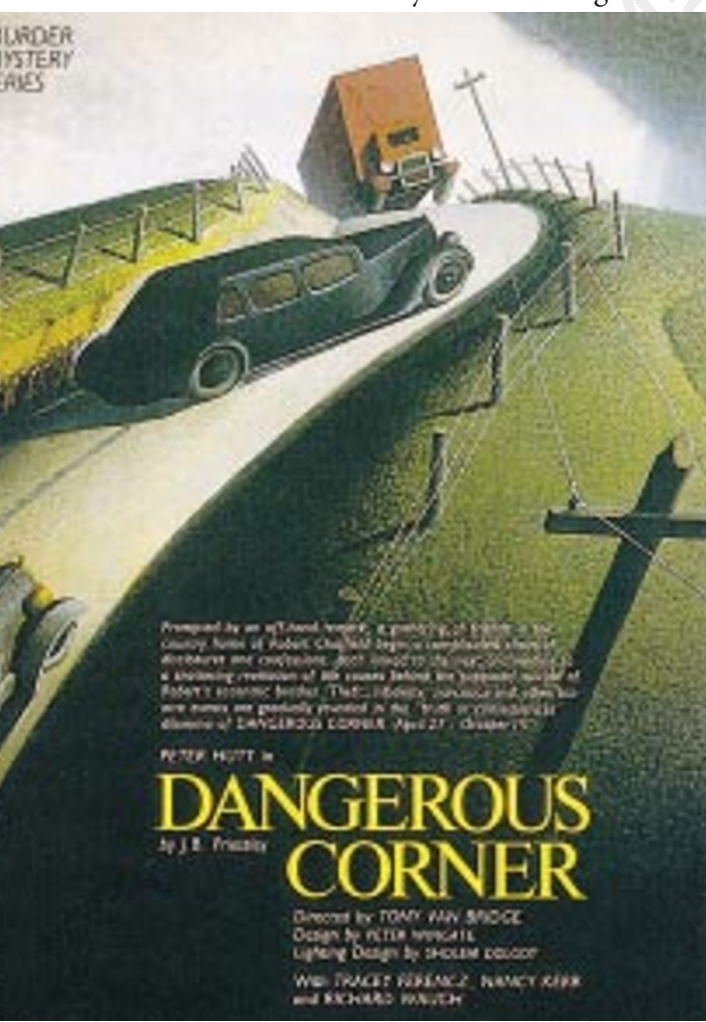

J. B. Priestley's 1932 play Dangerous Corner illustrates, says Derek York, the key concept of chaos - sensitivity to initial conditions. In 1964, Priestley also published a book entitled Man and Time. (From a 1988 Shaw Festival playbill.) poral axis, in the way described by the second law of thermodynamics. If Prigogine's methods do explain thermodynamic irreversibility, they simply account for this asymmetry they do not give us objective flow.

Leaving flow to one side, what is it that actually needs to be explained about the asymmetry of thermodynamics? Ordinarily we say it is the fact that entropy increases. But whether entropy is increasing or decreasing depends on which we take to be the 'positive' direction of time. Reverse the scale on the graph, and an increase becomes a decrease.

Is one choice of scale objectively the right one? Is what we call the future objectively the sense of 'positive' time? If so, this objective fact must consist of something other than the thermodynamic asymmetry itself. Otherwise, asking why entropy increases is like asking why a snake's head is at the front end: it would be true more or less by definition.

So there is a dilemma for Prigogine. Either he needs to pull two rabbits out of the hat - one to account for the objective direction of time itself, and another to account for the thermodynamic asymmetry - or the puzzle about irreversibility is not what he thinks it is. It isn't that entropy increases per $s e$, but rather that it increases in one direction and decreases in the other. (Analogously, the real puzzle about snakes is that the two ends are so different.)

In the latter case, Prigogine's methods are surely being applied to the wrong task. He suggests that the sensitivity of chaotic systems to initial conditions introduces unpredictability into physics, and hence explains why entropy is bound to increase. But the sensitivity applies whether we start with what we call initial conditions and work 'forwards' or start with final conditions and work 'backwards'. The more chaos helps to convince us that entropy increase is inevitable in one direction, the more it ought to amaze us that we see decrease in the other direction (a fact that surely ought to give us pause about the reliability of the 'explanation' in the former case: how good is a method that gets things so badly wrong in one case out of a possible two?).

Boltzmann came this way in 1877 , noting that it was a consequence of his new statistical methods that entropy ought to be higher in the past as well as in the future. The real 'time paradox' is that entropy goes down in one direction, not that it goes up in the other. Boltzmann may not have had a satisfactory solution, but - unlike Prigogine, I think - he certainly saw the significance of the problem.

So Prigogine's criticism of Boltzmann seems misplaced. To my mind, it is also counterproductive. One of the hardest things in this field is to set off on the right foot — to decide just what needs explaining. Clear directions are hard to find, and The End of Certainty lives up to its name in an unintended way: it isn't to blame for current uncertainty, but seems likely to prolong it.
In Derek York's book, by contrast, lack of a clear direction turns out to be an advantage. In Search of Lost Time is an engaging ramble through diverse topics temporal, by a man who has been dating for 40 years and seems to have loved every minute of it. I wish he had provided more references for readers inclined to explore on their own, but otherwise this is a delightfullittle book.

Huw Price is at the School of Philosophy, University of Sydney, NSW, Australia 2006.

\section{Future present}

\section{Beyond Calculation: The Next Fifty Years of Computing}

edited by Peter J. Denning and Robert M. Metcalfe

Copernicus: 1997. Pp. 313. \$24.30, £16.95

\section{Martin Campbell-Kelly}

How is this for an opener? "By 2047 almost all information will be in cyberspace including a large percentage of knowledge and creative works. All information about physical objects, including humans, buildings, processes and organizations, will be online. This trend is both desirable and inevitable."

This statement comes from the opening essay of this book, written by Gordon Bell, perhaps the most influential computer architect of his generation, and James $\mathrm{N}$. Gray, a database guru, both now senior researchers at Microsoft. Not many computer scientists or Web-surfers would dispute any of this, and it sets the tone for a compelling book. But don't be misled by the opening: what sets the book apart from the general run of technology-future books is the authority of its contributors and the tone of restraint that pervades it, when compared with the genre's usual ludicrous extrapolations.

For example, in the same essay Bell and Gray remind us that, despite the plummeting cost per bit and ever-increasing capacity of storage media, "paper is likely to be with us for ever... a lasting, irreplaceable graphical user interface. We know of no technology in 1997 to attack paper's broad use." What is more, they point out, acid-free paper has a longevity of more than 500 years, hundreds more than any known magnetic or optical storage medium.

Beyond Calculation is the edited proceedings of a conference organized in 1997 to celebrate the golden jubilee of the Association of Computing Machinery, the professional society of US information processing. The theme of the conference, as indicated by the book's title, is that computers have already gone far beyond numerical calculation, and have vastly further to go in the next half century. The conference organizers have done a fine job in assembling a star cast of leading computer technology researchers 
and people on the fringe of computing. The 20 essays in this fin de siècle collection have been seamlessly edited, and only three or four of the papers are evident pot-boilers.

The book is divided into three parts, dealing respectively with technology, computing and human identity, and business and economic issues. Of the six chapters in the technology stream, one of the highlights is an essay by Mark Weiser and John Seely Brown, both senior researchers at the famous Xerox PARC in Palo Alto, California. In the 1970s PARC researchers came up with the graphical user interface that 15 years later, as Microsoft Windows, made Bill Gates's fortune. Now workers at PARC have moved on to what they call "calm technology" - when computers become invisible and disappear into the woodwork. They foresee "clocks that find out the correct time after a power failure, kids' toys that are ever-refreshed with new software and vocabularies, paint that cleans off dust and notifies you of intruders, and walls that selectively dampen sounds." Got all that, Bill?

The first of the six essays on computers and human identity is by the eminent social researcher Sherry Turkle, who reflects on what it means for kids to grow up in a culture of video games and simulation. "Fifty years ago," she reminds us, "a child's world was full of things that could be understood in simple, mechanical ways. A bicycle could be understood in terms of its pedals and gears and a wind-up car in terms of its clockwork springs.” But lever the back off a Gameboy, and the child is confronted by an impenetrable chip. Thus children are led to psychological rather than mechanical explanations of their playthings; in effect, they perceive machines as having a kind of inner life "more alive than a car, but less alive than a bacterium".

Another essayist on computers and human identity is Terry Winograd, once enfant terrible of artificial intelligence, but now with his feet planted firmly on the floor. His essay touches on the vexed issue of the boundaries of computer science. Today there are terrific tensions and frustrations in computing research because a researcher on interface design probably has more in common with social psychologists on the other side of the campus than with the computer theoreticians in the same building. Winograd supposes that there may eventually be a split between interface designers and computer scientists, rather like the complementary roles of architect and civil engineer.

The final set of essays deals with economic and business issues. I think everyone will take away from this book a particular vision that captures their imagination above the others. For me it was an essay on "Information Warfare" by Larry Druffel, former director of the Software Engineering Institute. We have all picked up the idea, from the popular science press or voices in the air, that a new form of warfare is on the horizon. In this new blitzkrieg, taking out the enemy's information systems has the potential to do vastly more damage than the physical destruction caused by Second World War bombers. What would these weapons look like? One example Druffel cites is that of a 'rogue' program that floods the networks with bogus messages, impeding the free flow of information. The more advanced the economy, the greater the potential for devastation. Take out Albania's information systems and it probably wouldn't notice for three weeks. But do the same for the United States, 20 years down the road when it is totally dependent on the Internet, and the consequences would be awesome.

On a more upbeat note comes the delightful essay "There and Not There" by William Mitchell, the architect and cultural critic from the Massachusetts Institute of Technology, and Oliver Strimple, director of the Computer Museum in Boston. They ask why would anyone ever go out when everything they might want to see is on-line? In this articulate and optimistic essay, the authors persuasively argue that telepresence is no substitute for 'being there'. Television or a Web-browser simply cannot capture the full experience of going to a live pop concert, theatre performance, art gallery or soccer match. That indefinable, emotional something one gets at the real event they call 'aura'. We all knew it in our hearts, but they have given us a word for it.

Martin Campbell-Kelly is in the Department of Computer Science, University of Warwick,

Coventry CV4 7AL, UK.

\section{Incomplete theorems}

\section{What is Mathematics, Really?}

by Reuben Hersh

Oxford University Press/Cape: 1997. Pp. 343. $\$ 35, £ 18.99$

\section{Ivar Ekeland}

The title poses quite a question, and adding the word 'really' does not make it any easier. Empty words will not do: hard definitions are required. So what does 'really' really mean here?

It could mean: "What is modern mathematics, what makes it important in - or irrelevant to - our everyday life, what is it like to be an active mathematician?" There is much to say here. There has been an exponential growth in the number of scholars, books, journals, papers and theorems produced in the past century. Whole fields, such as probability theory, functional and numerical analysis, and noncommutative geometry, have been created. Older fields, such as algebraic

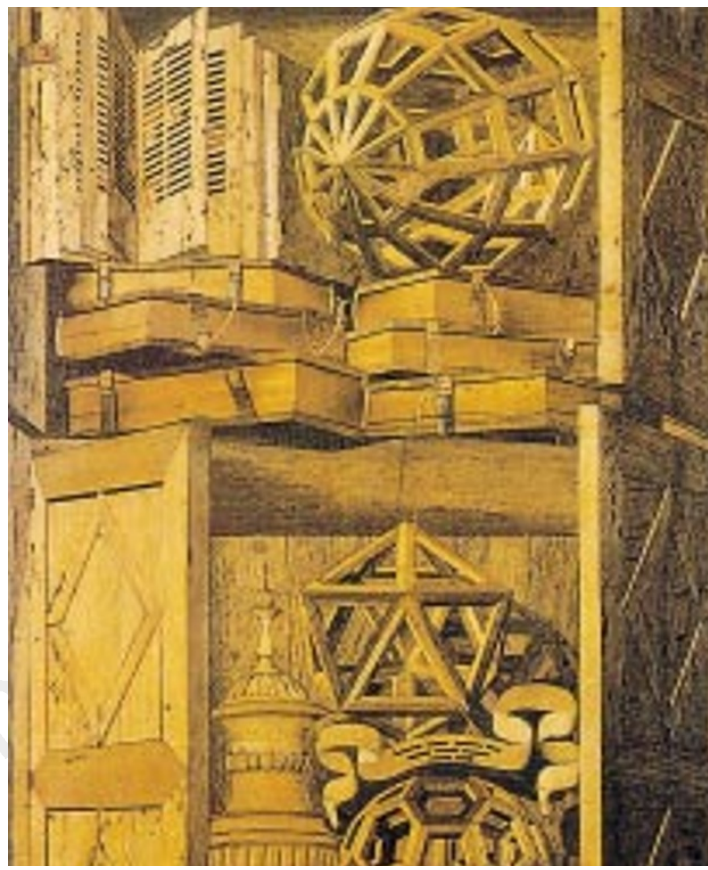

Facets of history: details of a wooden tarsia in the church of Santa Maria in Organo, Verona, Italy. From Polyhedra by Peter R. Cromwell

(Cambridge University Press, $\mathfrak{E} 30, \$ 44.95$ ).

geometry or number theory, have enjoyed tremendous progress, the solution of Fermat's last theorem being a case in point. The use of mathematics has become pervasive in engineering for filtering noisy signals and controlling processes, as well as in economics or finance, as shown by the success of the Black-Scholes model for pricing derivative securities.

It could also mean: "What is the inner meaning of mathematics?" Is there anything more to mathematics than its outward success story? Is it an activity to be pursued for its own sake, independently of material rewards? Painters or writers are supposed to be aiming for timeless achievement in art, not for recognition among their contemporaries, which may not even come during their lifetime. Is it the same with mathematicians? Are they artists in their own way? And, if so, what kind of art is theirs?

Strangely enough, this book goes neither way. It describes nothing of modern mathematics, except for a discussion about the foundations of mathematics and its connection with logic, which is far from the preoccupations of most working mathematicians. There is nothing at all to suggest the breadth and scope of mathematics today, or how much it has changed in the past century. It is always annoying to see the same tired, age-old examples crop up again and again: how to prove that the square root of two is irrational, how to use the tools of differentiation to exploit Galileo's law of falling bodies, and so on.

The book fails also to answer the question about the nature of mathematics. It dismisses it as "futile", leaving to forthcoming progress 